\title{
Quality of Life after Different Types of Ileal Diversions Following Radical Cystectomy
}

\author{
Mahmoud Abdel Hameed Mahmoud, Mohamed Abd Allah Hindawy, El Sayed Mohamed Hathout. \\ Urology Department, Faculty of Medicine, Al Azhar University \\ *Corresponding author: Mahmoud Abdel Hameed Mahmoud, Mobile: (+20) 01007953352, E-Mail: \\ drma7moud1991@gmail.com
}

\begin{abstract}
Background: Radical cystectomy remains the gold standard in treatment of muscle invasive bladder cancer. Objective: To evaluate the quality of life (QOL) for patients underwent ileal conduit (IC) versus orthotopic ileal neobladder (INB) urinary diversions (UD) after one year post radical cystectomy (RC).

Patients and methods: Our study was a retrospective study that included patients with MIBC who underwent RC and UD from January 2014 to Julie 2018 that passed one-year post RC. There were two groups of patients as follows: IC and INB groups. QOL was evaluated by the Functional Assessment of Cancer Therapy- Bladder Cancer (FACT-BL). The erectile function (EF) was assessed by the International Index of Erectile Function-5 (IIEF-5) questionnaire.

Results: Overall, 125 patients underwent RC with UD were screened for eligibility. Twenty patients (16\%) died, 11 patients $(8.8 \%)$ failed to reach to them, 6 patients $(4.8 \%)$ underwent other UD and 2 patients $(1.6 \%)$ refused participation in this study thus 86 patients $(68.8 \%)$ were eligible for the study and were analyzed. The mean patient's age was $61.6 \pm 6.3$ in both groups ( $\left.p_{\text {value }} 0.199\right)$. There were significant differences between both groups in physical, social, emotional, functional, additional concerns, urinary and GIT symptoms that were significantly better in INB group ( $\left.\mathrm{p}_{\text {value }}<0.001\right)$. There was no significant difference between both groups regarding the EF.

Conclusions: the INB is better than the IC UD in most of QOL domains, which include physical, social, emotional, functional, additional concerns, urinary and GIT symptoms. But sexual function remains equal bad in both groups.
\end{abstract}

Keywords: Urinary diversion, Quality of life, Radical cystectomy

\section{Introduction}

Bladder cancer (BC) is the seventh most commonly diagnosed cancer in the male population worldwide, while it drops to eleventh when both genders are considered, the worldwide age-standardized incidence rate (per 100,000 person/years) is 9.0 for men and 2.2 for women ${ }^{(1)}$. According to the first published national population-based study for the period between 2008 and 2011, BC is the 3rd most common malignancy $(6.9 \%)$ in Egypt with more predominance in males $78 \%$ than females $22 \%{ }^{(2)}$.

For patients with clinical T2-T4a, N0, M0 disease, Radical cystectomy (RC) and bilateral pelvic lymph node dissection remains the gold standard therapy by which all other treatment modalities should be compared. RC provides excellent local control with pelvic recurrence rates as low as $4 \%$ in patients with node negative disease ${ }^{(3)}$. The choice of the suitable UD technique depends on multiple factors including the patient age, associated co-morbidities, continence status, renal function, surgical margin and surgeon experience (4).
Various methods are used for UD after RC, including ileal conduits (IC), cutaneous continent urinary diversion, and orthotopic neobladder (INB) reconstruction ${ }^{(5)}$. The type of UD after RC has a great impact on different aspects of QOL including physical, sexual, psychosocial, day life activities and distress related to body image ${ }^{(6)}$. The concept of QOL differs significantly between cultures, countries, and races ${ }^{(7)}$.

There are multiple questionnaires available to measure QOL. These questionnaires can be general or disease-specific. Bladder specific questionnaires include functional assessment of cancer therapy for patients with bladder cancer (FACT-BL), European Organization for Research and Treatment of Cancer Core Quality of Life Questionnaire (EORTC-QLQ)-30 and Bladder Cancer Index (BCI) questionnaires ${ }^{(8)}$.

\section{Aim of the work:}

To evaluate the quality of life (QOL) for patients underwent ileal conduit (IC) versus orthotopic ileal 
neobladder (INB) urinary diversions (UD) after one year post radical cystectomy (RC).

\section{Patients and methods}

Our study was a retrospective study that included patients with MIBC who underwent RC and UD from January 2014 to Julie 2018 at Al Hussein and Bab El Sharia Al-Azhar University Hospitals that passed one-year post $\mathrm{RC}$ and were invited to participate in this study. The study sample was not limited by age, gender, or previous treatment.

\section{Ethical approval:}

The Research Ethics Committee of our institution approved the study protocol and all participants provided an informed consent before inclusion. There were two groups of patients as follows: IC UD group and INB UD group.

Data were retrospectively collected from the medical records regarding the demographic profile, body mass index (BMI), preoperative chemotherapy, preoperative co-morbidities, postoperative histopathology, pathological tumor stage (according to TNM staging system 2009) and postoperative complications.

Patients were excluded if they were indicated for palliative cystectomy, had a psychiatric disorder, cognitive morbidity (i.e. dementia, Alzheimer's disease), a history of alcohol or substance abuse, additional oncological disease, on dialysis, motor disabilities, refusal to participate in this study and incomplete follow-up data.

Two types of written questionnaire were used in assessment of the patients during their follow up interview that filled face to face by the doctors. Some others were telephoned to complete their answer. FACT-BL to determine their QOL and the IIEF-5 questionnaire to assess the EF of the males.

The FACT-BL is a validated questionnaire to Arabic version ${ }^{(9)}$. This designed for BC. It included 27-item of the FACT-general (G) questionnaire. This divided into 4 domains: physical, social, emotional, and functional wellbeing. An additional 12 urology-specific items (10 items related to gastrointestinal, urinary, sexual symptoms and 2 questions for patients with diversion) are included. Each item is scored from 0 "not at all" to 4 " very much" with higher scores indicating better QOL ${ }^{(8)}$.

The IIEF-5 questionnaire ${ }^{(\mathbf{1 0})}$, which was validated also to Arabic version (11) was used to assess recovery of sexual function in men after RC. The cutoff scores for normal erectile function are 25 and 21 , respectively with lower scores categorized as mild, moderate, and severe ED.

\section{Statistical analysis:}

Data management and statistical analysis were performed using the Statistical Package for Social Sciences (SPSS) version 24. Numerical data were summarized using means and standard deviations or medians and ranges. Data were explored for normality using Kolmogrov-Smirnov test and Shapiro-Wilk test. Categorical data were summarized as percentages. Comparisons between the 2 groups with respect to normally distributed numeric variables were done using the independent t-test. Non normally distributed numeric variables were compared by Mann-Whitney test. For categorical variables, differences were analyzed with $\chi^{2}$ (chi square) test and Fisher's exact test when appropriate. All p-values are two-sided. Pvalue $\leq 0.05$ were considered significant.

\section{Results}

Overall, 125 patients who underwent RC with UD were screened for eligibility. Twenty patients (16\%) died, 11 patients $(8.8 \%)$ failed to reach to them, 6 patients $(4.8 \%)$ underwent UD other than ileal diversion and 2 patients (1.6\%) refused participation in this study thus 86 patients (68.8\%) were eligible for the study and were analyzed.

The 86 patients were represented as two groups: IC 46 patients $(53.5 \%)$ and INB 40 patients $(46.5 \%)$. The mean observation time was $28.9 \pm$ 12.6 months. The mean (SD) patient's age was 61.6 \pm 6.3 years old and was statistically insignificant between both groups ( $\mathrm{p}$ value $=0.199$ ). Also, there was no significant difference between both groups in terms of sex, body mass index (BMI), educational status, pathological tumors stage, preand post- operative chemo \& radio-therapy (Table 1). Also, post-operative complications (Table 2). Table 3 showed the mean differences in QOL variables between both groups. There were significant differences between both groups in physical, social, emotional, functional and additional concerns. That were significantly better in INB group ( $\mathrm{P}$ value $<0.001)$.

As regard the urinary and GIT symptoms scale scores, there were significant difference in urinary incontinent, frequency of micturation and good appetite between both groups which were significantly better in patients with INB. But there was no significant difference in burning of micturation, diarrhea and control of bowel between both groups. Regarding like appearance of the body image scale score, it was significantly better in INB group than IC group as shown in table (4). 
Regarding the sexual function there was no significant difference in the degree of ED between both groups (Table 5).

Table (1): Patient's data and tumor characteristics.

\begin{tabular}{|c|c|c|c|}
\hline Variable & $\begin{array}{l}\text { Ileal conduit } \\
(\mathrm{n}=46) \\
\text { No. }(\%)\end{array}$ & $\begin{array}{c}\text { Ileal neo bladder } \\
(\mathrm{n}=\mathbf{4 0}) \\
\text { No. }(\%)\end{array}$ & P.value \\
\hline Age (years) & $62.4 \pm 5.8$ & $60.7 \pm 6.8$ & 0.199 \\
\hline BMI $\left(\mathrm{Kg} / \mathbf{m}^{2}\right)$ & $25.3 \pm 3.0$ & $25.6 \pm 2.2$ & 0.575 \\
\hline $\begin{array}{l}\text { Sex } \\
\quad \text { Male } \\
\text { Female }\end{array}$ & $\begin{array}{r}39(84.8) \\
7(15.2)\end{array}$ & $\begin{array}{r}36(90.0) \\
4(10.0)\end{array}$ & 0.470 \\
\hline $\begin{array}{l}\text { Educational status } \\
\text { Educated } \\
\text { Not educated } \\
\end{array}$ & $\begin{array}{l}11(23.9) \\
35(76.1) \\
\end{array}$ & $\begin{array}{l}14(35.0) \\
26(65.0) \\
\end{array}$ & 0.259 \\
\hline $\begin{array}{l}\text { Special habits } \\
\text { Smoker } \\
\text { Not }\end{array}$ & $\begin{array}{r}39(84.8) \\
7(15.2)\end{array}$ & $\begin{array}{r}35(87.5) \\
5(12.5)\end{array}$ & 0.717 \\
\hline $\begin{array}{l}\text { Histopathology } \\
\text { TCC. } \\
\text { SCC. }\end{array}$ & $\begin{array}{l}36(78.3) \\
10(21.7)\end{array}$ & $\begin{array}{l}29(72.5) \\
11(27.5)\end{array}$ & 0.535 \\
\hline $\begin{array}{l}\text { Pathological tumor stage } \\
\text { Organ confined }(\leq \mathrm{pT} 2, \mathrm{pN} 0) \\
\text { Non organ confined (pT3- pT4a, pN0) } \\
\text { lymph node-positive (pN }+ \text { ) }\end{array}$ & $\begin{array}{c}20(43.5) \\
20(43.5) \\
6(13)\end{array}$ & $\begin{array}{c}19(47.5) \\
15(37.5) \\
6(15)\end{array}$ & 0.638 \\
\hline $\begin{array}{l}\text { Chemotherapy } \\
\text { No } \\
\text { Yes }\end{array}$ & $\begin{array}{c}42(91.3) \\
4(8.7)\end{array}$ & $\begin{array}{r}32(80) \\
8(20)\end{array}$ & 0.131 \\
\hline $\begin{array}{l}\text { Radiotherapy } \\
\text { No } \\
\text { Yes }\end{array}$ & $\begin{array}{r}41(89.1) \\
5(10.9)\end{array}$ & $\begin{array}{c}34(85.0) \\
6(15)\end{array}$ & 0.567 \\
\hline
\end{tabular}

BMI: body mass index, TCC: transitional cell carcinoma, SCC: squamous cell carcinoma P.value $\leq 0.05$ is considered statistically significant.

Table (2): post-operative complication

\begin{tabular}{|c|c|c|c|c|c|c|}
\hline Variables & & Ileal & Conduit & Ileal & Neo bladder & P.value \\
\hline & & No. & $(\%)$ & No. & $(\%)$ & \\
\hline Early post- & Yes & 28 & 60.9 & 26 & 65 & 0.693 \\
\hline operative & No & 18 & 39.1 & 14 & 35 & \\
\hline \multirow[t]{5}{*}{ complications } & Wound infection & 10 & 21.7 & 9 & 22.5 & 0.932 \\
\hline & Wound dehiscence & 3 & 6.5 & 3 & 7.5 & 1.000 \\
\hline & Blood transfusion & 11 & 23.9 & 14 & 35 & 0.259 \\
\hline & Ileus & 4 & 8.7 & 3 & 7.5 & 1.000 \\
\hline & Metabolic acidosis & 4 & 8.7 & 4 & 10 & 1.000 \\
\hline Late post- & Yes & 10 & 21.8 & 14 & 35 & 0.171 \\
\hline operative & No & 36 & 78.2 & 26 & 65 & \\
\hline \multirow[t]{4}{*}{ complications } & Incisional hernia & 5 & 10.9 & 5 & 12.5 & 1.000 \\
\hline & $\begin{array}{l}\text { Deterioration of renal } \\
\text { function }\end{array}$ & 5 & 10.9 & 7 & 17.5 & 0.759 \\
\hline & DVT & - & - & 1 & 2.5 & 0.475 \\
\hline & Uretero-pouchal stenosis & - & - & 1 & 2.5 & 0.475 \\
\hline
\end{tabular}

DVT: deep venous thrombosis

Table (3): FACT-BL scores in both groups. 
ejhm.journals.ekb.eg

\begin{tabular}{|l|c|c|c|}
\hline \multicolumn{1}{|c|}{ Variables } & & $95 \%$ CI & \\
\cline { 2 - 4 } & Ileal conduit & Ileal neo bladder & p.value \\
\hline Physical well-being & $17.6 \pm 3.2$ & $25.5 \pm 2.0$ & $<\mathbf{0 . 0 0 1} *$ \\
\hline Social well-being & $19.2 \pm 1.9$ & $23.7 \pm 1.3$ & $<\mathbf{0 . 0 0 1} *$ \\
\hline Emotional well-being & $16.9 \pm 2.4$ & $22.1 \pm 1.2$ & $<\mathbf{0 . 0 0 1} *$ \\
\hline Functional well-being & $18.9 \pm 1.7$ & $23.9 \pm 1.5$ & $<\mathbf{0 . 0 0 1} *$ \\
\hline Additional concerns & $27.0 \pm 3.8$ & $31.5 \pm 2.3$ & $<\mathbf{0 . 0 0 1} *$ \\
\hline FACT-BL & $105.0 \pm 10.2$ & $134.4 \pm 6.3$ & $<\mathbf{0 . 0 0 1} *$ \\
\hline
\end{tabular}

CI: confidence interval *: statistically significant differences (p.value $<0.05)$

Table (4): FACT-BL scores between both groups as regard urinary, GIT and like appearance of the body symptoms.

\begin{tabular}{|l|c|c|c|c|c|c|c|}
\hline \multirow{2}{*}{ Variables } & \multicolumn{3}{|c|}{ Ileal conduit } & \multicolumn{3}{c|}{ Ileal neo bladder } & \multicolumn{1}{c|}{} \\
\cline { 2 - 9 } & Med. & Min. & Max. & Med. & Min. & Max. & p.value \\
\hline Urinary incontinent & 1 & 1 & 2 & 3 & 2 & 4 & $<\mathbf{0 . 0 0 1 *}$ \\
\hline Control of bowel & 4 & 3 & 4 & 4 & 2 & 4 & 0.699 \\
\hline Frequency of micturation & 2 & 1 & 3 & 4 & 3 & 4 & $<\mathbf{0 . 0 0 1} *$ \\
\hline Diarrhea & 4 & 1 & 4 & 4 & 2 & 4 & 0.100 \\
\hline Appetite & 3 & 1 & 4 & 4 & 3 & 4 & $<\mathbf{0 . 0 0 1 *}$ \\
\hline Burning micturation & 4 & 2 & 4 & 4 & 3 & 4 & 0.104 \\
\hline Like appearance of body image & 1 & 1 & 2 & 4 & 3 & 4 & $<\mathbf{0 . 0 0 1 *}$ \\
\hline
\end{tabular}

Med.: median Min.: minimum Max.: maximum *: statistically significant differences (p.value <0.05).

Table (5): degree of ED using of the IIEF-5 Questionnaire

\begin{tabular}{|l|c|c|c|}
\hline Variables & $\begin{array}{c}\text { Ileal conduit } \\
(\mathbf{n = 3 9 )} \\
\text { No. }(\boldsymbol{\%})\end{array}$ & $\begin{array}{c}\text { Ileal neo bladder } \\
(\mathbf{n = 3 6}) \\
\text { No. }(\boldsymbol{\%})\end{array}$ & p.value \\
\hline Sever ED & $24(61.5)$ & $15(41.7)$ & \\
\hline Moderate ED & $10(25.6)$ & $11(30.6)$ & 0.260 \\
\hline Mild to moderate ED & $3(7.7)$ & $4(11.1)$ & \\
\hline Mild ED & $2(5.1)$ & $6(16.7)$ & \\
\hline
\end{tabular}

ED: erectile dysfunction.

\section{Discussion}

The gold standard treatment for MIBC is RC with UD. Types of UD have a great impact on different aspects of QOL including physical, social, functional, micturation status, sexual, psychosocial functioning, day life activities and distress related to body image ${ }^{(12)}$.

There are many limitations to achieve comparability between QOL results in different studies such as multiple questionnaires are used to measure the QOL in oncological urology ${ }^{(13)}$. Not all the questionnaires have been validated, they differ in some of the topics covered. QOL is incorporating different domains that may change over time, between different cultures ${ }^{(\mathbf{1 4})}$.

So, we used the validated FACT-BL questionnaire in order to exclude as many of the abovementioned problems as possible. In the present study, analysis of FACT-BL questionnaire showed that patients with INB diversion were significantly better than those with IC diversion in the assessment of the general health status and QOL including physical, social, emotional and functional domains. The emotional well- being also remained significant higher in ileal neobladder group than ileal conduit even after many years. These results are consistent with those obtained by Hobisch et al. (15) using the QLQ-C30 questionnaire. Philip et al. ${ }^{(16)}$ and Fujisawa et al. (17) using the Short-Form-36 questionnaire found that patients who underwent continent reconstruction had significantly better physical functioning than those who underwent incontinent diversion. Erber et al. ${ }^{(\mathbf{1 8})}$ reported a better QOL in patients with an INB diversion. Moeen et al. ${ }^{(19)}$ using the same FACT-BL questionnaire, found that there were significant differences between both groups in the physical, social/family, emotional, functional and additional concerns that were better in INB group. "Emotional well-being" became statistically insignificant one year postoperatively while other parameters remained significantly better in INB group even after the first year. Only Gilbert et al. ${ }^{(20)}$ reported that patients with INB have higher distress due to urinary leakage. 
In the present study we assessed the QOL after one-year post- operative as at this time the general condition of the patients become more stabilized. Kulaksizoglu et al. ${ }^{(21)}$ discussed the appropriate time to assess the QOL after UD. They measured it before and at different intervals after surgery. They found that the psychological and QOL scores decreased at the initial postoperative period and came back to the baseline level and stabilized at 12 months after RC. So, they recommended that QOL measurement should not to be done before one year after RC.

Concerning other symptom scale scores in the present study, there was significant difference in urinary incontinence, frequency of micturation, good appetite and like appearance of the body that were significantly higher in INB group than IC group but no significant difference was found in burning micturation, control of bowel symptoms and the diarrhea. Erber et al. ${ }^{(18)}$ found a higher incidence of diarrhea postoperatively $(\mathrm{P}=0.004)$ in patients who had undergone INB diversion than in those with IC diversion. This might be due to a large segment of bowel used in INB reconstruction leading to an increased incidence of diarrhea postoperative but became stabilized at one-year postoperative ${ }^{(18)}$. As regards like appearance of the body symptom scale score, it was significantly better in the INB group than the IC group due to altered body image and the presence of the collecting system in the ileal conduit group that limits the daily activities of the patients due to urine leakage and odor.

In the present study, out of 75 male patients ED affected $89.3 \%$. Also, there was no significant difference between the degree of ED and the type of the UD (both groups were affected). The occurrence of erection is the most affected pattern, even if occurred become not firm enough to start the intercourse. In addition to iatrogenic ED in men after RC, other factors might impair sexual function such as changes in body image, the emotional and the psychological responses of patients and their partners. Moreover, the stigma associated with UD might impair sexuality and sexual satisfaction. After RC there is a change in sexual function for both sexes and ED can affect up to $80 \%$ of men, which is an important QOL issue (22). Allareddy et al. ${ }^{(23)}$ reported that $89 \%$ of patients who were treated by RC could not maintain erection compared to $32 \%$ of patients not treated by RC. Even among men who regain some EF, erections become not firm enough for intercourse. Hedgepeth et al. (24) reported improvement in sexual function in patients managed with ONB in contrast to those managed by IC.

The main limitation of this study lies in lack of pre-operative QOL assessment. And also, in the retrospective design. Obviously perioperative patient care and methods of patient counseling have been steadily improved and changed now and might be affected our results. Due to the retrospective nature of our study based on two heterogeneous cohorts, prospective randomized trials would be needed to prove real value. The strength of the present study lies in the fact of avoidance of the selection bias where patient's preoperative health status, (or some other factors) may have been different before cystectomy, and this might be affected the choice of diversion.

\section{Conclusion:}

In conclusion RC and UD are a major surgery affecting all aspect of the QOL. How QOL will be affected is an important point of an ongoing intense research. Although ileal neobladder forms may provide better QOL, this may be statistically insignificant. The QOL may not be only affected by the type of UD but also by age of the patient, method of the operation and postoperative complications.

\section{References}

1. Antoni S, Ferlay J, Soerjomataram I et al. (2017): Jemal A, Bray F. Bladder cancer incidence and mortality: a global overview and recent trends. European Urology, 71 (1): 96-108.

2. Ibrahim AS, Khaled HM, Mikhail NN et al. (2014): Cancer incidence in egypt: results of the national population-based cancer registry program. J Cancer Epidemiol., 437971: 18.

3. Herring JC, Kamat AM (2004): Treatment of muscle-invasive bladder cancer: progress and new challenges. Expert Review of Anticancer Therapy, 4 (6): 1047-56.

4. Sugihara T, Yasunaga H, Horiguchi $\mathrm{H}$ et al. (2014): Factors affecting choice between ureterostomy, ileal conduit and continent reservoir after radical cystectomy: Japanese series. Int J Clin Oncol., 19 (6): 1098-104.

5. Stenzl A, Sherif H, Kuczyk M (2010): Radical cystectomy with orthotopic neobladder for invasive bladder cancer: A critical analysis of long term oncological, functional and quality of life results. Int Braz J Urol., 36: 537-47. 
6. Gerharz EW, Mansson A, Hunt S et al. (2005): Quality of life after cystectomy and urinary diversion: an evidence-based analysis. J Urol., 174: 1729-1736.

7. Svare J, Walter S, Kvist Kristensen J et al. (1985): Ileal conduit urinary diversion: Early and late complications. Eur Urol., 11: 83-6.

8. Gilbert SM, Dunn RL, Hollenbeck BK (2010): Development and validation of the Bladder Cancer Index: a comprehensive, disease specific measure of health-related quality of life in patients with localized bladder cancer. J Urol., 183: 1764-70.

9. Zahran MH, Sheir K, Zidan EM et al. (2017): Validation of the Arabic version of the Functional Assessment of Cancer Therapy-Bladder questionnaire in Egyptian patients with bladder cancer. Arab Journal of Urology, 15 (2): 1104.

10. Rosen RC, Riley A, Wagner $\mathrm{G}$ et al. (1997): The international index of erectile dysfunction: multidimensional scale for assessment of erectile dysfunction. Urology, 49: 822830.

11. Shamloul R, Ghanem H, Abou-Zeid A (2004): Validity of the Arabic version of the sexual health inventory for men among Egyptians. International Journal of Impotence Research, 16 (5): 452-6.

12. Montie JE, Clark PE, Eisenberger MA et al. (2009): bladder cancer. J Natl Compr Canc Netw., 7 (1): 8-39.

13. Dombo O, Otto U (2005): Lebensqualit at nach radikal-chirurgischen urologischen Eingriffen im Becken und die Bedeutung der Rehabilitation. Urologe., 44 (1): 1128.

14. Wright JI, Porter MP (2007): Quality-oflife assessment in patients with bladder cancer. Nature Clinical Practice Urology, 4 (3): 147-154.

15. Hobisch A, Tosun $K$, Kinzl $J$ et al. (2000): Quality of life after cystectomy and orthotopic neobladder versus ileal conduit urinary diversion. World J Urol., 18: 338-44.

16. Philip J, Manikandan R, Venugopal S et al. (2009): Orthotopic neobladder versus ileal conduit urinary diversion after cystectomy: A quality-of-life based comparison. Ann R Coll Surg Engl., 91: 565-9. 47.

17. Fujisawa M, Isotani S, Gotoh A et al. (2000): Health related quality of life with orthotopic neobladder versus ileal conduit according to the SF-36 survey. Urology, 55: 862- 5 .

18. Erber B, Schrader M, Miller $\mathrm{K}$ et al. (2012): Morbidity and quality of life in bladder cancer patients following cystectomy and urinary diversion: a singleinstitution comparison of ileal conduit versus orthotopic neobladder. ISRN Urol., ISRN Urol. ,2012:342796.

19. Moeen AM, Safwat AS, Gadelmoula MM et al. (2018): Health related quality of life after urinary diversion. Which technique is better? Journal of the Egyptian National Cancer Institute, 30 (3): 93-7.

20. Gilbert SM, Dunn RL, Hollenbeck BK (2010): Development and validation of the Bladder Cancer Index: a comprehensive, disease specific measure of health-related quality of life in patients with localized bladder cancer. J Urol., 183:1764-70.

21. Kulaksizoglu H, Toktas G, Kulaksizoglu IB et al. (2002): When should quality of life be measured after radical cystectomy? Eur Urol., 42:350-5.

22. Modh RA, Mulhall JP, Gilbert SM (2014): Sexual dysfunction following cystectomy and urinary diversion. Nat Rev Uro., 11: 445-53.

23. Allareddy V, Kennedy J, West MM et al. (2006): Quality of life in long-term survivors of bladder cancer. Cancer, 106: 2355-62.

24. Hedgepeth RC, Gilbert SM, He C et al. (2010): Body image and bladder cancer specific quality of life in patients with ileal conduit and neobladder urinary diversions. Urology, 5: 763-671. 